\title{
Collapse of Non-axisymmetric Cavities
}

\author{
Oscar R. Enriquez, Ivo R. Peters, Stephan Gekle, Laura Schmidt, \\ Michel Versluis, Devaraj van der Meer, and Detlef Lohse \\ Physics of Fluids Group \\ University of Twente, The Netherlands
}

March 3, 2022

\begin{abstract}
A round disk with a harmonic disturbance impacts on a water surface and creates a non-axisymmetric cavity which collapses under the influence of hydrostatic pressure. We use disks deformed with mode $\mathrm{m}=2$ to $\mathrm{m}=6$. For all mode numbers we find clear evidence for a phase inversion of the cavity wall during the collapse. We present a fluid dynamics video showing high speed imaging of different modes, pointing out the characteristic features during collapse.
\end{abstract}

Web version (9.4 MB)

Full quality version (221 MB) 